\title{
Dilepton $p_{\mathrm{T}}$ suppression in the color glass condensate
}

\author{
M.A. Betemps, M.B. Gay Ducati ${ }^{\mathrm{a}}$ \\ High Energy Physics Phenomenology Group, GFPAE, Instituto de Física, Universidade Federal do Rio Grande do Sul, Caixa \\ Postal 15051, CEP 91501-970, Porto Alegre, RS, Brazil
}

Received: 21 January 2005 / Revised version: 19 April 2005 /

Published online: 8 July 2005 - (C) Springer-Verlag / Società Italiana di Fisica 2005

\begin{abstract}
Dilepton production is investigated in proton-nucleus collisions in the forward region using the color glass condensate approach. The transverse momentum distribution $\left(p_{\mathrm{T}}\right)$, more precisely the ratio between proton-nucleus and proton-proton differential cross section for LHC energies is evaluated, showing the effects of saturation at small $p_{\mathrm{T}}$, and presenting a suppression of the Cronin type peak at moderate $p_{\mathrm{T}}$. These features indicate the dilepton as the most suitable probe to study the properties of the saturated regime and the Cronin effect in these systems.
\end{abstract}

PACS. 11.15.Kc, 24.85.+p

\section{Introduction}

The search for signatures of a color glass condensate (CGC) description of the saturated regime is an outstanding aspect of investigation in heavy ion colliders. The first results of the Relativistic Heavy Ion Collider (RHIC), on charged hadron multiplicity in $\mathrm{Au}-\mathrm{Au}$ collisions, were treated considering that the CGC formulation gives a natural qualitative explanation of the data [1]. However, there are several issues to be clarified before concluding that the dynamical of the partonic system should be described by a CGC already at RHIC energies.

In this work we investigate quantitative features of the dilepton production in the forward region of protonnucleus collisions, since $p A$ collisions were proposed as an ideal experiment to study the saturation effects described by the CGC in the forward rapidity [2]. In particular, the transverse momentum $\left(p_{\mathrm{T}}\right)$ distribution is studied focusing attention to the small $p_{\mathrm{T}}$ region, where the saturation effects are expected to be more significant and considered as a possible signature of the saturation effects when contrasted with proton-proton results. This comparison is performed evaluating the ratio between proton-nucleus and proton-proton cross section. This ratio shows two distinct behaviors: it presents a Cronin type peak (if a local Gaussian for the correlator function is used), and a large suppression (if a non-local Gaussian is used), being a most suitable probe of the status of the Cronin effect as a final or initial state effect.

\footnotetext{
a e-mail: gay@if.ufrgs.br
}

\section{The color glass condensate}

The color glass condensate picture holds in a frame in which the hadron propagates at the speed of light and, by Lorentz contraction, appears as an infinitesimally thin two-dimensional sheet located at the light cone.

At small $x$ and/or large $A$ one expects the transition of the regime described by the standard perturbative QCD [DGLAP and BFKL] to a new regime where processes like recombination of partons should be important in the parton cascade [3,4]. In this regime, the growth of the parton distribution is expected to saturate below a specific scale $Q_{\mathrm{s}}[5]$, forming a color glass condensate [6-9]. This saturated field, meaning the dominant field of gluons, has a large occupation number and allows for the use of semiclassical methods. These methods provide the description of the small $x$ gluons as being radiated from fast moving color sources (parton with larger values of $x$ ), being described by a color source density $\rho_{a}$, with internal dynamics frozen by Lorentz time dilatation, thus forming a color glass. The color fields are driven by the classical Yang-Mills equation of motion with the sources given by the large $x$ partons. The large $x$ partons move nearly at the speed of light in the positive $z$ direction.

In the CGC approach light cone variables are employed $\left(x^{\mu} \equiv\left(x^{+}, x^{-}, x_{\perp}\right)\right)$. The large $x$ partons (fast) have momentum $p^{+}$, emitting (or absorbing) soft gluons with momentum $k^{+} \ll p^{+}$, generating a color current only with the + component $J_{a}^{+}=\delta\left(x^{-}\right) \rho_{a}$. In order to have a gaugeinvariant formulation, the source $\rho_{a}$ must be treated as a stochastic variable with zero expectation value. An average on configurations is required being performed through a weight function $W_{A^{+}}[\rho]$, which depends upon the dynamics of the fast modes, and upon the intermediate scale $\Lambda^{+}$, which defines fast $\left(p^{+}>\Lambda^{+}\right)$and soft $\left(p^{+}<\Lambda^{+}\right)$ 
modes. Any physical observable is obtained by averaging the solution to the classical Yang-Mills equation over all configurations of $\rho_{a}$, with the gauge-invariant weight function $W_{\Lambda^{+}}[\rho]$.

The effective theory is valid only at soft momenta of order $\Lambda^{+}$. Indeed, going to a much softer scale, there are large radiative corrections which invalidate the classical approximation. The modifications to the effective classical theory are governed by a functional, non-linear, evolution equation, the JIMWLK equation $[7,8]$, for the statistical weight function $W_{\Lambda^{+}}[\rho]$ associated with the random variable $\rho_{a}(x)$.

The solution for such a functional evolution equation is not well determined yet and in order to make predictions or a comparison with the data, some phenomenological treatment should be given to the weight function. In this work an approximation to the weight function which is reasonable when we have large nuclei is used and consists in taking a Gaussian form [10-12]. In [11] it is shown that a Gaussian weight function can accommodate both the BFKL evolution of the gluon distribution at high transverse momenta, and the gluon saturation phenomenon at low transverse momenta. A non-local Gaussian distribution of color sources has been constructed in [13] as a mean-field asymptotic solution for the JIMWLK equation and provides some modifications concerning phenomenological properties of the observables $[14,15]$. In the following sections the phenomenological consequences of the choice of a local or non-local Gaussian type for the weight function are investigated concerning the transverse momentum of the dileptons.

\section{Dilepton production in the CGC approach}

At high energies, the dilepton production in hadronic collisions looks like a bremsstrahlung of a virtual photon with the momentum $\boldsymbol{p}$ decaying into a lepton pair [16,17]. This can occur before and after the interaction of the quark (momentum $\boldsymbol{k}$ ) with the dense saturated gluonic field (momentum $\boldsymbol{q}$ ) of the target, in our case the nucleus $A$. We consider only the diagrams where the photon emission occurs before the interaction or after the interaction with the nucleus. Calculation considering the emission both before and after the interaction leads to vanishing values [18].

In order to obtain a hadronic cross section, the validity of the collinear factorization in the fragmentation region is assumed and the cross section reads [15]

$$
\begin{aligned}
& \frac{\mathrm{d} \sigma^{p A \rightarrow q l^{+} l^{-} X}}{\mathrm{~d} p_{\mathrm{T}}^{2} \mathrm{~d} M \mathrm{~d} x_{\mathrm{F}}}=\frac{4 \pi^{2}}{M} R_{A}^{2} \frac{\alpha_{\mathrm{em}}^{2}}{3 \pi} \frac{1}{x_{1}+x_{2}} \\
& \times \int \frac{\mathrm{d} l_{\mathrm{T}}}{(2 \pi)^{3}} l_{\mathrm{T}} W\left(p_{\mathrm{T}}, l_{\mathrm{T}}, x_{1}\right) C\left(l_{\mathrm{T}}, x_{2}, A\right),
\end{aligned}
$$

where $x_{\mathrm{F}}$ is the longitudinal momentum fraction given by $x_{\mathrm{F}}=x_{1}-x_{2}$, and $x_{1}$ and $x_{2}$ are the momentum fraction carried by the quark from the proton and by the gluonic field from the nucleus, respectively. The expression (1) is valid in the forward region, which means positive $x_{\mathrm{F}}$, or positive rapidities $y$, and the variables $x_{1}$ and $x_{2}$ are defined by

$$
x_{\left(\begin{array}{l}
1 \\
2
\end{array}\right)}=\frac{1}{2}\left\{\sqrt{x_{\mathrm{F}}+4 \frac{M_{\mathrm{T}}^{2}}{s}}( \pm) x_{\mathrm{F}}\right\},
$$

where $M_{\mathrm{T}}^{2}=M^{2}+p_{\mathrm{T}}^{2}$ is the squared dilepton transverse mass and $s$ is the squared center of mass energy. Here, using the structure function $F_{2}\left(x, Q^{2}\right)=\sum_{i} e_{q_{i}}^{2} x\left[q_{i}\left(x, Q^{2}\right)+\right.$ $\left.\bar{q}_{i}\left(x, Q^{2}\right)\right]$, the weight function $W\left(p_{\mathrm{T}}, l_{\mathrm{T}}, x_{1}\right)$ can be written as $[18]$

$$
\begin{aligned}
& W\left(p_{\mathrm{T}}, l_{\mathrm{T}}, x_{1}\right)=\int_{x_{1}}^{1} \mathrm{~d} z z F_{2}\left(x_{1} / z, M^{2}\right) \\
& \times\left\{\frac{\left(1+(1-z)^{2}\right) z^{2} l_{\mathrm{T}}^{2}}{\left[p_{\mathrm{T}}^{2}+M^{2}(1-z)\right]\left[\left(p_{\mathrm{T}}-z l_{\mathrm{T}}\right)^{2}+M^{2}(1-z)\right]}\right. \\
& -z(1-z) M^{2}\left[\frac{1}{\left[p_{\mathrm{T}}^{2}+M^{2}(1-z)\right]}\right. \\
& \left.\left.\quad-\frac{1}{\left[\left(p_{\mathrm{T}}-z l_{\mathrm{T}}\right)^{2}+M^{2}(1-z)\right]}\right]^{2}\right\} .
\end{aligned}
$$

Such a weight function selects the values of $l_{\mathrm{T}}$ larger than $p_{\mathrm{T}} . R_{A}$ is the nuclear radius. $z \equiv p^{-} / k^{-}$is the energy fraction of the proton carried by the virtual photon, $l_{\mathrm{T}}=$ $q_{\mathrm{T}}+p_{\mathrm{T}}$ is the total transverse momentum transfer between the nucleus and the quark. The function $C\left(l_{\mathrm{T}}\right)$ is the field correlator function and defined by [12]

$$
C\left(l_{\mathrm{T}}\right) \equiv \int \mathrm{d}^{2} x_{\perp} \mathrm{e}^{\mathrm{i} l_{\mathrm{T}} \cdot x_{\perp}}\left\langle U(0) U^{\dagger}\left(x_{\perp}\right)\right\rangle_{\rho},
$$

with the averaged term representing the average over all configurations of the color fields in the nucleus, and $U\left(x_{\perp}\right)$ is a matrix in the $S U(N)$ fundamental representation which represents the interactions of the quark with the classical color field. All the information about the nature of the medium crossed by the quark is contained in the function $C\left(l_{\mathrm{T}}\right)$. In particular, it determines the dependence on the saturation scale $Q_{\mathrm{s}}$ (and on energy). In our calculations the CTEQ6L parametrization [19] was used for the structure function, and the lepton pair mass gives the scale for the projectile quark distribution.

In (1) one includes an energy dependence in the field correlator function only in the saturation scale $Q_{\mathrm{s}, A} \rightarrow$ $Q_{\mathrm{s}, A}(x)$ to simulate a low $x$ evolution, in order to investigate the effects of the $x$ evolution in the dilepton $p_{\mathrm{T}}$ spectra. The $x$ dependence is parametrized in the form proposed by Golec-Biernat and Wüsthoff (GBW) [20] $\left(Q_{\mathrm{s}}^{2}=\left(x_{0} / x\right)^{\lambda}\right)$, with the parameters taken from the dipole cross section extracted from the fit procedure by CGCfit [21] parametrizations, which will be discussed later. The nuclear radius, which appears in (1), is taken from the Woods-Saxon parametrization, which has the form, $R_{A}=1.2 A^{1 / 3} \mathrm{fm}$, while the proton radius (when calculating $p p$ collisions) is taken from the fits.

The effect of saturation is encoded in the function $C\left(l_{\mathrm{T}}\right)$ at the small $l_{\mathrm{T}}$ region. The behavior of the weight function $W\left(p_{\mathrm{T}}, l_{\mathrm{T}}, x_{1}\right)$ determines that only at small $p_{\mathrm{T}}$ 
the effects of saturation in the function $C\left(l_{\mathrm{T}}, x, A\right)$ should be measurable, once the weight function selects the values of $l_{\mathrm{T}}$ larger than $p_{\mathrm{T}}[15]$.

To make a quantitative prediction to the dilepton production, the correlator function $C\left(l_{\mathrm{T}}, x, A\right)$ has to be determined. It plays an important role in the color glass condensate formalism and will be discussed in the next section.

\section{The color field correlator $C\left(l_{\mathrm{T}}, x, A\right)$}

The function $C\left(l_{\mathrm{T}}\right)$ is considered a fundamental quantity in the CGC formalism, since it contains all the information on high density effects. Considering the MV model, the function $C\left(l_{\mathrm{T}}\right)$ should be computed in the form [15]

$$
\begin{aligned}
& C_{M V_{\text {mod }}}\left(l_{\mathrm{T}}, x, A\right) \\
& =\int \mathrm{d}^{2} x_{\perp} \mathrm{e}^{\mathrm{i} l_{\mathrm{T}} \cdot x_{\perp}} \mathrm{e}^{-\frac{Q_{\mathrm{S}}^{2}(x, A)}{\pi} \int \frac{\mathrm{d} p}{p^{3}}\left(1-J_{0}\left(p x_{\perp}\right)\right)},
\end{aligned}
$$

where we have introduced the dependence on the energy and nuclei into the saturation scale. The nuclear saturation scale is parametrized in the form

$$
Q_{\mathrm{s}}^{2}(x, A)=A^{1 / 3} Q_{\mathrm{s}}^{2}(x) .
$$

The field correlator function presented up to here in MV model is obtained considering a local Gaussian function for the weight function $W_{\Lambda^{+}}[\rho]$. The local Gaussian appears in the definition of the correlator function (3) in the averaged term $\left\langle U(0) U^{\dagger}\left(x_{\perp}\right)\right\rangle_{\rho}$. The consideration of a non-local Gaussian function modifies the correlator in such a way that it is written as $[11,14]$

$$
C\left(l_{\mathrm{T}}, x, A\right) \equiv \int \mathrm{d}^{2} x_{\perp} \mathrm{e}^{\mathrm{i} l_{\mathrm{T}} \cdot x_{\perp}} \mathrm{e}^{\chi\left(x, x_{\perp}, A\right)},
$$

with

$$
\begin{aligned}
& \chi\left(x, x_{\perp}, A\right) \\
& \equiv-\frac{2}{\gamma c} \int \frac{\mathrm{d} p}{p}\left(1-J_{0}\left(x_{\perp} p\right)\right) \ln \left(1+\left(\frac{Q_{2}^{2}(x, A)}{p^{2}}\right)^{\gamma}\right),
\end{aligned}
$$

where $\gamma$ is the anomalous dimension $(\gamma \approx 0.64$ for BFKL) and $c \approx 4.84[11,14]$.

The physical effect of the nonlocal Gaussian weight function is that the gluon sources are no longer correlated locally, as in the local Gaussian, but correlate over larger distances. This implies in a more drastic reduction of the gluon density at small $l_{\mathrm{T}}$. The effect of the local or nonlocal Gaussian weight function in the $p_{\mathrm{T}}$ dilepton spectra will be discussed in the next section in the context of the defined ratio $R_{p A}$.

Having addressed all the fundamental aspects to develop the calculation of the dilepton transverse momentum in the CGC formalism, one presents in the next section the numerical predictions using such an approach and the discussions.

\section{Results and discussions}

In what follows, the numerical results on the dilepton transverse momentum distribution in CGC are addressed and discussed. In the original work [15] we consider $p A$ collisions at maximum projected RHIC $(\sqrt{s}=350 \mathrm{GeV})$ and LHC energies $(\sqrt{s}=8.8 \mathrm{TeV})$ in the proton fragmentation region (positive rapidities). Here we employ only LHC energies. The calculations are performed fixing values of rapidities (or $x_{\mathrm{F}}$ ) and lepton pair mass $M$. We use the function $C\left(l_{\mathrm{T}}, x_{2}\right)$ based on the MV model, however an $x$ dependence through the saturation scale is introduced, taking the parameters from [21].

In order to avoid any ambiguity with normalization, the ratio between the proton-nucleus and proton-proton differential cross section is defined,

$$
R_{p A}=\frac{\frac{\mathrm{d} \sigma(p A)}{\pi R_{A}^{2} \mathrm{~d} M \mathrm{~d} x_{\mathrm{F}} \mathrm{d} p_{\mathrm{T}}^{2}}}{A^{1 / 3} \frac{\mathrm{d} \sigma(p p)}{\pi R_{p}^{2} \mathrm{~d} M \mathrm{~d} x_{\mathrm{F}} \mathrm{d} p_{\mathrm{T}}^{2}}} .
$$

Some attention should be given to the uncertainty in the determination of the nuclear radius, then each cross section is divided by the nuclear or proton radius. The factor $A^{1 / 3}$ was used in the denominator to guarantee a ratio $R_{p A}$ about 1 at large $p_{\mathrm{T}}$. By definition, the ratio in (8) is similar to the one obtained in the [14] to investigate the Cronin effect.

The Cronin effect was discovered in the late's 70s [22] and is related to the enhancement of the hadron transverse momentum spectra at moderated $p_{\mathrm{T}}(2-5 \mathrm{GeV})$ in comparison with the proton-proton collisions (the ratio between $p A$ and $p p$ presents a peak at moderate $p_{\mathrm{T}}$ ). The effect should be interpreted as being originated by the multiple scatterings of the partons from the proton propagating through the nucleus, resulting in a broadening of the transverse momentum of the initial partons. The Cronin effect was recently measured by the RHIC experiments, in $\mathrm{Au}-\mathrm{Au}$ and $d-\mathrm{Au}$ collisions, and presents a different behavior: the suppression of the peak is found and is claimed to be due to final state interactions [23]. Although the Cronin effect was observed in the hadron transverse momentum spectra, it can be also analyzed in the dilepton transverse momentum spectra, since multiple scatterings are expected as an initial state effect. The dilepton $p_{\mathrm{T}}$ spectra should clarify the properties of the Cronin effect, once no final state interactions occur in this observable. Moreover, the ratio obtained in this work is similar to that used to investigate the Cronin effect [14].

In Fig. 1 one presents the results for the ratio $R_{p A}$ to LHC energies considering a correlator field function $C\left(l_{\mathrm{T}}, x, A\right)$ obtained from a local and non-local Gaussian distribution for the weight function $W_{\Lambda^{+}}[\rho]$. The solid line represents the calculation for rapidity $y=2.2$ and local Gaussian; the dashed line for rapidity $y=3.2$ and local Gaussian. It is verified that at moderate $p_{\mathrm{T}}$ the calculations show a Cronin type peak. However, if a non-local Gaussian is used the suppression of the peak is reached, which is shown in the long-dashed line to rapidity 2.2 and by the dot-dashed line to rapidity 3.2 . 


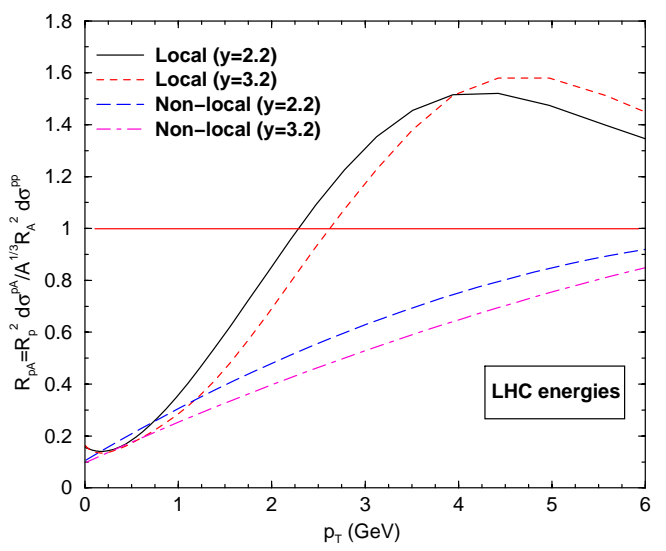

Fig. 1. Ratio between proton-nucleus and proton-proton at LHC energies by the CGC approach at distinct rapidities with local and non-local Gaussian distribution for the weight function $W[\rho]$

The more recent experimental data on Cronin effects present the suppression of the peak $[24,25]$. If the local Gaussian correlator function with the same energy dependence as implemented here is used [14], the CGC predicts a Cronin peak at intermediated $p_{\mathrm{T}}$ in complete disagreement with the BRAHMS experiment at forward rapidities $[24,25]$. In the same reference [14] the Cronin effect was studied using a non-local Gaussian distribution for the weight function and the Cronin peak suppression is reached. In the dilepton sector, using the non-local Gaussian, the suppression of the ratio $R_{p A}$ is verified showing exactly the same features presented in the Cronin effect [14], being a possible clean observable to study the properties of this effect. Although, the Cronin effect was considered as a final state one in [26], in our analysis, the dilepton production seems to clarify this aspect. It was obtained that the Cronin type peak (or the suppression of the Cronin peak) in the dilepton $p_{\mathrm{T}}$ distribution appears as an initial state effect.

At LHC energies, the suppression of the ratio $R_{p} A$ reaches large values of $p_{\mathrm{T}}$ and such a suppression increases with the rapidity. It is interesting to address the fact that at LHC the experimental facilities provide a detection of dileptons in the forward region with transverse momentum above $1.5 \mathrm{GeV}$, depending on the rate of the signal of the observable and on the signal from physics and machine sources [27]. This feature assures that at LHC energies such a suppression behavior should be detectable.

\section{Conclusions}

In this work the large saturation effects described by the color glass condensate in the dilepton production at small $p_{\mathrm{T}}$ region are calculated. The comparison between $p p$ and $p A$ cross section, provides a tool to study the Cronin effect and the dynamics of the color glass condensate.

Particularly, the dilepton transverse momentum distribution presents the suppression of the Cronin type peak, as observed in the inclusive observables, if a non-local Gaussian is used. Such a behavior is observed in Fig. 1. At forward rapidities at the LHC energies, the effect of suppression increases with the rapidity. Such a large suppression at high energies gives an indication that the dilepton transverse momentum distribution provides a clear probe of the color glass condensate description of the high energy hadronic interactions in the forward rapidity region.

Acknowledgements. MBGD would like to thank the organizers for the invitation to this exciting conference. This work was partially supported by CNPq, Brazil.

\section{References}

1. D. Kharzeev, E. Levin, Phys. Lett. B 523, 79 (2001); D. Kharzeev, E. Levin, L. McLerran, Phys. Lett. B 561, 93 (2003)

2. Y.V. Kovchegov, A.H. Mueller, Nucl. Phys. B 529, 451 (1998)

3. L.V. Gribov, E.M. Levin, M.G. Ryskin, Phys. Rep. 100, 1 (1983)

4. A.L. Ayala, M.B. Gay Ducati, E.M. Levin, Nucl. Phys. B 493, 305 (1997); B 511, 355 (1998)

5. A.L. Ayala, M.B. Gay Ducati, E.M. Levin, Phys. Lett. B 388, 188 (1996)

6. L. McLerran, R. Venugopalan, Phys. Rev. D 49, 2233 (1994); D 49, 3352 (1994)

7. J. Jalilian-Marian, A. Kovner, A. Leonidov, H. Weigert, Nucl. Phys. B 504, 415 (1997); Phys. Rev. D 59, 014014 (1999)

8. E. Iancu, A. Leonidov, L.D. McLerran, Nucl. Phys. A 692, 583 (2001); Phys. Lett. B 510, 133 (2001)

9. E. Ferreiro, E. Iancu, A. Leonidov, L. McLerran, Nucl. Phys. A 703, 489 (2002)

10. E. Iancu, R. Venugopalan, hep-ph/0303204

11. E. Iancu, K. Itakura, L. McLerran, Nucl. Phys. A 724, 181 (2003)

12. F. Gelis, A. Peshier, Nucl. Phys. A 697, 879 (2002)

13. E. Iancu, K. Itakura, L. McLerran, Nucl. Phys. A 724, 181 (2003)

14. J.P. Blaizot, F. Gelis, R. Venugopalan, Nucl. Phys. A 743, 13 (2004)

15. M.A. Betemps, M.B. Gay Ducati, Phys. Rev. D 70, 116005 (2004)

16. B.Z. Kopeliovich, in Proceedings Workshop Hirschegg'95: Dynamical Properties of Hadrons in Nuclear Matter, edited by H. Feldmeier, W. Nörenberg, GSI, Darmstadt, p. 102 (1995) [hep-ph/9609385]; S.J. Brodsky, A. Hebecker, E. Quack, Phys. Rev. D 55, 2584 (1997)

17. B.Z. Kopeliovich, J. Raufeisen, A.V. Tarasov, Phys. Lett. B 503, 91 (2001)

18. F. Gelis, J. Jalilian-Marian, Phys. Rev. D 66, 094014 (2002)

19. J. Pumplin, D.R. Stump, J. Huston, H.L. Lai, P. Nadolsky, W.K. Tung, JHEP 0207, 012 (2002)

20. K. Golec-Biernat, M. Wüsthoff, Phys. Rev. D 59, 014017 (1999); D 60, 114023 (1999); Eur. Phys. J C 20, 313 (2001)

21. E. Iancu, K. Itakura, S. Munier, Phys. Lett. B 590, 199 (2004) 
M.A. Betemps, M.B. Gay Ducati: Dilepton $\boldsymbol{p}_{\mathbf{T}}$ suppression in the color glass condensate

22. J.W. Cronin et al., Phys. Rev. D 11, 3105 (1975)

23. A. Accardi, nucl-th/0405046

24. BRAHMS Collaboration, R. Debbe, J. Phys. G 30, S759 (2004)
25. BRAHMS Collaboration, I. Arsene et al., Phys. Rev. Lett. 93, 242303 (2004) nucl-ex/0403005

26. R.C. Hwa, C.B. Yang, Phys. Rev. Lett. 93, 082302 (2004)

27. ALICE Collaboration, CERN/LHCC-99-22, August 1999; K. Safarik, private communication 